\title{
Risk of type 2 diabetes mellitus in women with prior hypertensive disorders of pregnancy: a systematic review and meta-analysis
}

\author{
Grace Zhao ${ }^{1}$ (D) $\cdot$ Dominika Bhatia $^{2}$ (D) $\cdot$ Flora Jung $^{1}$ (D) $\cdot$ Lorraine Lipscombe $^{2,3}$ (D)
}

Received: 12 July 2020 / Accepted: 9 October 2020 / Published online: 7 January 2021

(C) Springer-Verlag GmbH Germany, part of Springer Nature 2021

\begin{abstract}
Aims/hypothesis The association between a history of hypertensive disorders of pregnancy (HDP) and subsequent type 2 diabetes (referred to throughout as diabetes) remains inconclusive. We reviewed the most recent evidence to quantify the association of previous HDP with incident diabetes.

Methods A systematic search of MEDLINE, Embase and CINAHL was performed up to 17 February 2020 to identify observational studies of the association between HDP (pre-eclampsia or gestational hypertension) and incident diabetes. Studies of women with pre-pregnancy diabetes were excluded. Two independent reviewers screened citations and abstracted results. Study quality was assessed in duplicate using the Newcastle-Ottawa Scale. Random-effects models were used to pool effect estimates. Heterogeneity was assessed using the $I^{2}$ statistic.

Results After screening 4617 citations, 16 cohort studies with a total of 3,095,457 participants were included (unspecified HDP $n=5$, pre-eclampsia only $n=4$, gestational hypertension and pre-eclampsia $n=7$ ). Risks of subsequent diabetes were significantly higher in women with a history of any HDP (HDP: adjusted hazard ratio [aHR] 2.24, 95\% CI 1.95, 2.58; gestational hypertension: aHR 2.19 [95\% CI 1.69, 2.84]; pre-eclampsia: aHR 2.56 [95\% CI 2.02, 3.24]; preterm pre-eclampsia: aHR 3.05 [95\% CI 2.05, 4.56]). The association between HDP and diabetes persisted in studies that adjusted for gestational diabetes mellitus (aHR 2.01 [95\% CI 1.77, 2.28]).

Conclusions/interpretation HDP are independently associated with a higher risk of diabetes. Further study is needed to determine how HDP contribute to diabetes risk prediction to develop evidence-based screening and prevention strategies.
\end{abstract}

Keywords Diabetes $\cdot$ Gestational hypertension $\cdot$ Hypertensive disorders of pregnancy $\cdot$ Meta-analysis $\cdot$ Pre-eclampsia Systematic review

\begin{abstract}
Abbreviations
aHR Adjusted hazard ratio

GDM Gestational diabetes mellitus

HDP Hypertensive disorders of pregnancy

ICD International Classification of Diseases

NOS Newcastle-Ottawa Scale
\end{abstract}

Grace Zhao

grace.zhao@mail.utoronto.ca

1 MD Program, Faculty of Medicine, University of Toronto, Toronto, ON, Canada

2 Institute of Health Policy, Management and Evaluation, Dalla Lana School of Public Health, University of Toronto, Toronto, ON, Canada

3 Women's College Research Institute, Women's College Hospital, Toronto, ON, Canada

\section{Introduction}

Hypertensive disorders of pregnancy (HDP) affect approximately $15 \%$ of parous women and are among the leading causes of maternal and perinatal mortality worldwide [1-3].

HDP are classified into pre-existing (chronic) hypertension, gestational hypertension, pre-eclampsia/eclampsia, and pre-eclampsia superimposed on chronic hypertension [4]. For our study, HDP included gestational hypertension and preeclampsia, including HELLP (haemolysis, elevated liver enzymes, lowered platelets) syndrome and eclampsia.

Women with HDP are more often hyperinsulinaemic than those with normotensive pregnancies [5] and may exhibit insulin resistance during pregnancy that is independent of obesity and glucose intolerance [6, 7]. HDP may thus be an early marker of type 2 diabetes (referred to throughout as diabetes) risk based on shared metabolic risk factors. It has also been postulated that systemic inflammation associated with pre-eclampsia may damage the maternal vascular 


\section{Research in context}

\section{What is already known about this subject?}

- Hypertensive disorders of pregnancy (HDP) are among the leading causes of maternal and perinatal mortality worldwide

- The association between previous HDP and type 2 diabetes remains inconclusive

- Current diabetes screening guidelines do not include HDP as an independent risk factor for diabetes

What is the key question?

- $\quad$ Are previous HDP independently associated with a higher subsequent risk of type 2 diabetes mellitus?

What are the new findings?

- Women with previous HDP had a twofold increased risk of developing type 2 diabetes later in life that was independent of $\mathrm{BMI}$ and the presence of gestational diabetes

- Women with previous HDP developed diabetes earlier than women with normotensive pregnancies, with the highest risk occurring within 20 years after the index pregnancy

- There was a dose-response relationship between HDP severity and diabetes incidence, with greatest risk observed in pre-eclampsia or preterm pre-eclampsia

\section{How might this impact on clinical practice in the foreseeable future?}

- This paper provides evidence that previous HDP are an independent risk factor for type 2 diabetes that may be used to risk-stratify women for postpartum screening and prevention. Prospective studies are needed to identify an optimal follow-up strategy targeted for women with a history of HDP that is cost-effective and patient centred

endothelium, further predisposing such women to develop diabetes and future CVD $[8,9]$. Indeed, several studies have suggested a link between HDP and an increased risk for diabetes later in life [10-12]. As the prevalence of diabetes continues to rise globally $[13,14]$, it is critical to identify atrisk populations that can be targeted for timely diabetes screening and prevention strategies. The increased metabolic demands and frequent healthcare contact of pregnancy provide a unique window of opportunity to identify and reduce risk of future disease.

It is well known that gestational diabetes mellitus (GDM) is an independent risk factor for the development of diabetes [12, 15-18], and guidelines currently recommend that all women with GDM be screened for diabetes and offered prevention counselling postpartum [16, 19, 20]. However, it remains to be determined how the presence of HDP influences future risk of diabetes, and whether postpartum diabetes screening guidelines should include women with HDP. Previous meta-analyses were limited by either: (1) potential confounding from comorbid GDM, pre-existing diabetes or chronic hypertension [21]; or (2) the small number of studies that did account for confounders [22], making it difficult to delineate the independent contribution of HDP to risk of diabetes. Several higher quality studies with longer followup times, larger sample sizes and more complete adjustment for pre-pregnancy confounders have since been published on the association between HDP and diabetes [23-27], which provide more clarity regarding the relationship between HDP and diabetes.

In this systematic review and meta-analysis, we aimed to review the most recent evidence to quantify the risk of incident diabetes in women with previous HDP independent of the presence of GDM. Our secondary aim was to estimate the impact of time since a pregnancy complicated by HDP and risk of diabetes. The findings of this study will help inform future recommendations on the influence of a history of HDP on diabetes risk stratification, screening and prevention for postpartum women.

\section{Methods}

The protocol was developed a priori in accordance with the Preferred Reporting Items for Systematic reviews and MetaAnalyses (PRISMA) guidelines (ESM Checklist 1) [28, 29]. The Meta-analysis Of Observational Studies in Epidemiology (MOOSE) reporting guidelines were followed (ESM Checklist 2) [30]. The present systematic review was prospectively registered with PROSPERO (registration ID CRD42020168399).

Search strategy We developed the search strategy in consultation with a health sciences information specialist and searched MEDLINE (Ovid), Embase (Ovid) and CINAHL 
(EBSCO) from inception to 17 February 2020 for cohort and case-control studies, for which a validated search filter was used [31]. Studies were restricted to the English language due to lack of professional translators. Electronic search terms included but were not limited to 'pregnancy-induced hypertension,' 'diabetes mellitus' and 'pre-eclampsia'. Reference lists of included studies and relevant reviews were screened to identify additional relevant studies. Authors were contacted for data clarification when necessary. The full search strategy can be found in ESM Table 1.

Eligibility criteria and outcomes Study eligibility was defined using the following PECO (population, exposure, comparison group, outcome) criteria: (1) parous women; (2) included HDP as a predictor; (3) included a comparison group of women without HDP; and (4) reported risk estimates of type 2 diabetes later in life as an outcome. Studies that did not explicitly exclude women with pre-gestational diabetes (type 1 or 2) at baseline were excluded. Similarly, we excluded studies that did not account for the presence of chronic hypertension at baseline by way of exclusion, stratification or multivariable analysis; however, studies where chronic hypertension status was not explicit were kept in our review and handled in subgroup analyses. Studies of women who reported hypertension within one year after their delivery were also excluded because this may represent previously undetected chronic hypertension. To capture the most robust original evidence that has undergone peer review, abstracts, conference proceedings, letters to the editor and literature reviews were excluded.

Study selection Abstracts and full texts were assessed for eligibility in duplicate by two independent reviewers (two of GZ, FJ, DB), with one reviewer (GZ) reviewing all abstracts and full texts. As recommended by the Cochrane Handbook for Systematic Reviews of Interventions [32], disagreements on study eligibility ( $<15 \%$ of the screened full-text sample) were discussed with the study team and resolved by consensus.

Data abstraction Data abstraction was performed by one reviewer (GZ) and verified by a second reviewer (DB). For included articles, GZ extracted study characteristics (i.e. year, country, study design), population characteristics, sample size, types of HDP studied, ascertainment of HDP and type 2 diabetes, whether the study focused on first pregnancy, confounding factors (i.e. pre-gestational diabetes, GDM, chronic hypertension, cardiovascular risk factors), exclusion of postpartum hypertension and diabetes ( $<6$ months postpartum), follow-up length from the index pregnancy (pregnancy used to mark the start of follow-up time to the onset of diabetes), age at type 2 diabetes diagnosis, incidence and risk estimates of type 2 diabetes subcategorised into gestational hypertension, pre-eclampsia, preterm pre-eclampsia (preeclampsia that resulted in preterm birth $<37$ weeks) and unspecified HDP (studies that did not specify the subtype of HDP), as well as adjustment factors.

Risk of bias Quality appraisals were performed by two reviewers (GZ, FJ) independently. Any discrepancies were resolved by discussion and involvement of a third independent reviewer (DB). As recommended by the Cochrane NonRandomized Studies Methods Group [33], we assessed the quality of cohort and case-control studies using the Newcastle-Ottawa Scale (NOS) [34]. The NOS allocates up to 9 points according to three quality domains: selection of study groups (4 points); comparability between the exposure and non-exposure group ( 2 points); and ascertainment of outcomes (3 points). For the 'comparability' domain, controlling for pre-pregnancy diabetes and chronic hypertension were deemed to be particularly important as these are known independent risk factors for diabetes. Studies deemed 'low risk' had a minimum of 3 points in the selection domain, 1 point in comparability and 2 points in the outcomes domain. Studies that had 2 points in the selection domain - at least 1 point in comparability and 2 points in outcomes-were classified as 'medium risk'. Studies that scored 0 in comparability or 1 in any other domain were deemed 'high risk'. Funnel plots were produced to assess the risk of publication bias. Visual inspection and Egger's test of the intercept were used to assess funnel plot asymmetry [32].

Statistical analysis Random-effects meta-analyses were performed to quantify the effect of HDP on the risk of developing subsequent type 2 diabetes. The random-effects method is preferable when there is significant heterogeneity, as it assumes the existence of a distribution of distinct but related exposure effects on the outcome [32]. This approach therefore reports the average exposure effect on the outcome [32]. Studies were combined using DerSimonian-Laird inverse variance method, which assigns weights to individual estimates based on their precision level $[32,35]$. Only the most adjusted study estimates were pooled to minimise confounding. The majority of studies reported adjusted hazard ratios (aHR). ORs can be thought to approximate RRs under the rare events assumption [36-38]. Although ORs/RRs are conceptually different from HRs [39], a decision was made to pool them with HRs, as their exclusion in the sensitivity analyses did not meaningfully modify the pooled estimates (ESM Table 2) [38]. If a study reported multiple unique effect size estimates, they were all entered into the meta-analyses as long as they represented mutually-exclusive subgroups of individuals. In studies of overlapping participant subgroups, the more representative and/or conservative estimate was chosen. Heterogeneity was assessed using the $I^{2}$ statistic, which describes the variability of effect size estimates attributable 
to study differences, rather than chance [32]. Heterogeneity was explored through subgroup and sensitivity analyses by HDP type, follow-up duration, study quality, and confounders accounted for by way of exclusion, stratification, or multivariable adjustment (i.e. BMI, cardiovascular risk factors, GDM, chronic hypertension), provided at least three unique effect size estimates were available [32]. Specifically, to assess the robustness of effect size estimates for the association between HDP and diabetes to confounding, we pooled studies that did and did not account for one of the key confounding factors above into separate subgroups. The influence of these factors was first considered individually and then together, to provide the most independent estimate of the effect of HDP on diabetes risk. All analyses were two-tailed and results were considered significant at $p<0.05$. All analyses were performed using Cochrane's Review Manager (RevMan), version 5.3 (Copenhagen, Denmark), and the 'meta', 'metafor' and 'dmetar' packages in RStudio, version 4.0.2.

\section{Results}

Study characteristics The systematic search yielded 4617 citations after duplicates were removed, of which 16 studies met our relevance screen (Fig. 1). Individual study characteristics are presented in Table 1.

The included 16 studies were published between 2007 and 2019, with 12 retrospective and four prospective cohort studies (Table 1). A total of 3,095,457 women were included, with a median age of 32 at delivery and median follow-up of 11 (range 1-40) years after the index pregnancy. Five studies examined unspecified HDP [10, 25-27, 40], four examined pre-eclampsia only [24, 41-43], four examined both gestational hypertension and pre-eclampsia [11, 12, 44, 45], and three examined HDP, gestational hypertension and preeclampsia $[23,46,47]$. Four studies focused on the first pregnancy $[23,24,42,44]$, three studies explicitly excluded postpartum hypertension $[11,23,45]$ and four excluded postpartum diabetes [11, 12, 26, 41]. About half were conducted in Europe $(n=7 ; 44 \%)$ and the remaining studies were from North America $(n=5 ; 31 \%)$, Asia $(n=3 ; 19 \%)$ and Australia $(n=1 ; 6 \%)$ (Table 1$)$. The confounding factors accounted for varied (Table 1), where 11 studies accounted for GDM [10-12, 25, 26, 40-42, 45-47] (six by stratification $[12,40-42,45,46])$, ten studies accounted for chronic hypertension through exclusion, stratification, or adjustment [11, $12,23,24,40-42,45-47]$ and seven studies adjusted for BMI $[10,11,23,25-27,40]$. The mean sample HDP prevalence was $21.5 \%$ for HDP, $9.1 \%$ for gestational hypertension and $12.7 \%$ for pre-eclampsia. Four studies reported ORs [10, $25,43,45]$ and the remaining 11 studies reported HRs [11, 12, $23,24,26,40-42,44,46,47]$. One study did not report a relative measure of effect size and thus was only included for the narrative synthesis [27].

Ascertainment of HDP Hypertensive disorders of pregnancy were determined using administrative data (registries, remuneration claims, insurance databases) in nine studies [12, 24, $25,41,42,44-47]$, self-report in five studies [23, 25-27, 40], medical records in two studies $[11,43]$ and consultant obstetricians in one study [10]. One self-reported definition was validated by medical records [23] and another medical record definition was validated by obstetricians [11]. For the studies that did include GDM $(n=11)$ [10-12, 25, 26, 40-42, 45-47], only one study specified that GDM was diagnosed by Carpenter and Coustan criteria [42]. In the remaining studies, GDM was ascertained in the same way as HDP, using International Classification of Diseases (ICD) diagnostic codes or self-report.

Ascertainment of diabetes Status was determined by administrative data in ten studies [11, 12, 24, 41-47] and self-report in six studies [10, 23, 25-27, 40]. Of these, six studies used ICD codes [11, 24, 42, 44, 46, 47], two used diabetes databases [12, 43], one used hospital discharge diagnoses [45], and another used prescription drugs as proxy for diabetes (insulin, biguanides, sulfonamides, alpha glucosidase inhibitors, thiazolidinediones, meglitinides) [41]. It should be noted that two self-reported outcomes also utilised laboratory results, glucose-lowering medications or lifestyle therapy when diabetes was not self-reported [25, 40]. One study that used administrative data confirmed the diagnosis through laboratory or pharmaceutical prescription records (metformin, sulfonylureas, thiazolidinediones, acarbose, miglitol, nateglinide, repaglinide, insulin) [42]. All but six studies $[12,24,41,42,45,47]$ had at least 10 years of follow-up after the index pregnancy.

Systematic review Women with prior HDP developed diabetes at earlier ages than women with normotensive pregnancies $[23,27]$. Many studies did not indicate the time interval from the index pregnancy to diabetes diagnosis, particularly if the study focused on midlife diabetes development $[25,26]$. Despite that, several studies indicated that for women with prior HDP, the greatest risk of diabetes occurred between ages 35 and 40 [12, 23, 42, 46, 47]. Stuart et al (2018) found that women with pre-eclampsia had a statistically significant 2.6fold increased risk of diabetes as early as 6-10 years after their first birth which continued throughout a 40-year follow-up period [23]. For women with gestational hypertension, this increased risk began 21-25 years after their first birth. Wang et al (2012) found the strongest risk of type 2 diabetes mellitus for women with HDP aged 35-40 with an adjusted HR of 8.87 $(95 \%$ CI $2.46,32.0)$ and an incidence of 87.3 per 10,000 person-years [47]. 
Fig. 1 PRISMA flow diagram of literature search and selection process (adapted from [28]). DM, diabetes mellitus
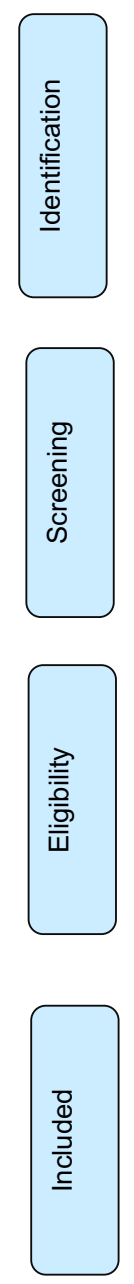

Records identified through database searching $(n=6499)$

Ovid MEDLINE: $n=1980$

Ovid Embase: $n=3911$

EBSCO CINAHL Plus: $n=608$

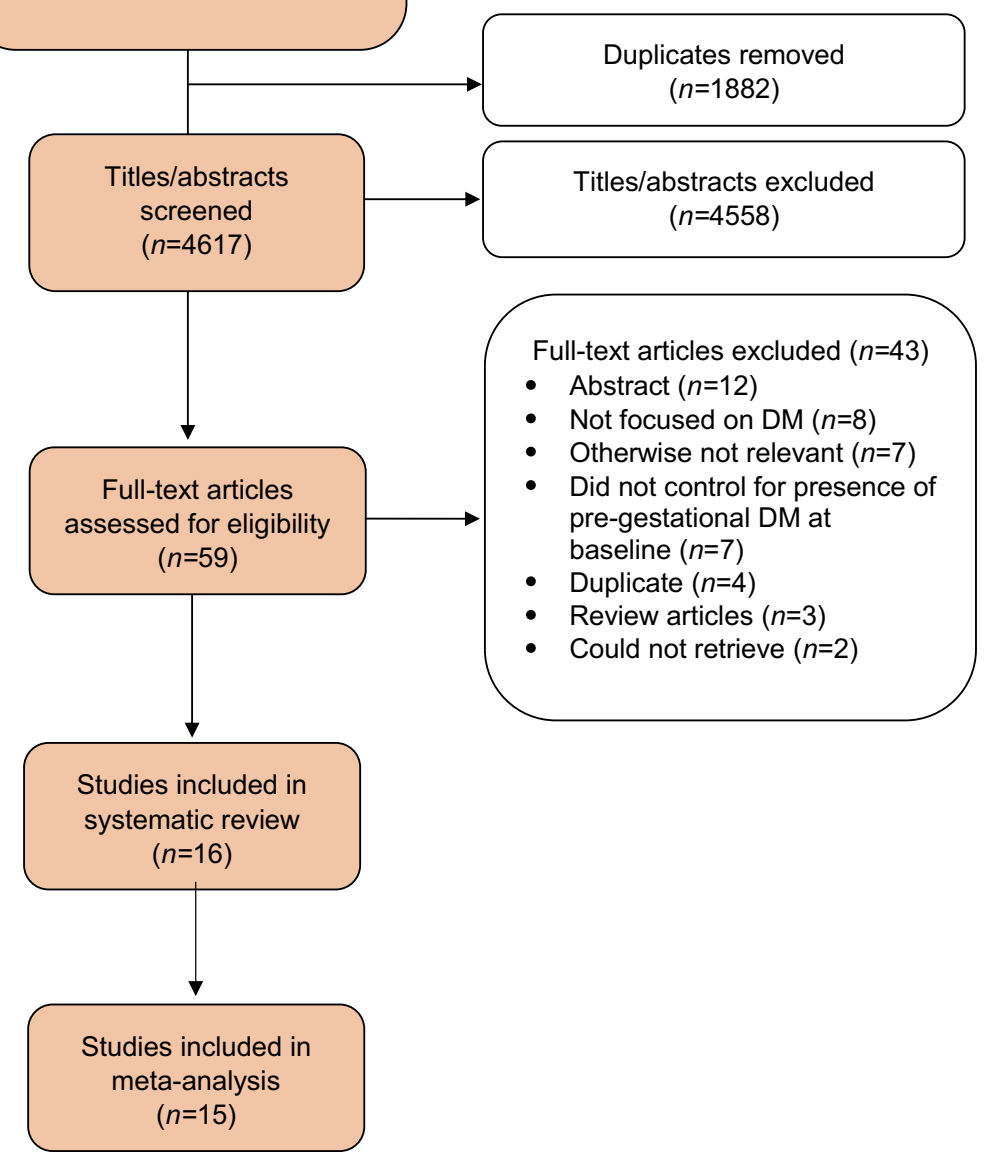

Interestingly, Timpka et al (2019) found a significant interaction between post-pregnancy BMI and HDP for the risk of diabetes later in life with the greatest additive effect for women with obesity [26]. However, across all BMI groups, women with HDP appeared to have a higher cumulative incidence of diabetes after age 50 .

Meta-analysis Fifteen studies were included in the meta-analysis, all of which had a cohort design (Fig. 2). Overall, the risk of diabetes in women with any prior HDP was 2.24 (95\% CI $1.95,2.58, I^{2}=94 \%$ ) relative to no HDP. We found a doseresponse relationship between HDP severity and diabetes risk, with pre-eclampsia/preterm pre-eclampsia having the greatest risk (gestational hypertension: aHR 2.19 [95\% CI 1.69, 2.84] $I^{2}=87 \%$; pre-eclampsia: aHR $2.56\left[95 \%\right.$ CI 2.02, 3.24] $I^{2}=$ 94\%; preterm pre-eclampsia: aHR 3.05 [95\% CI 2.05, 4.56] $I^{2}=82 \%$ ) (Table 2). The risk for diabetes associated with HDP remained elevated even among studies that accounted for the presence of GDM (aHR 2.01 [95\% CI 1.77, 2.28]).
Studies with a shorter follow-up duration (less than 20 years) showed a greater risk of diabetes than those with more extended follow-up (Table 2). Heterogeneity was somewhat explained by HDP type, types of confounders accounted for, follow-up duration and a focus on first pregnancy in subgroup analyses, but the overall magnitude of effect size for the association between HDP and diabetes remained robust (Table 2 and ESM Table 3). In studies that controlled for BMI, the association between HDP and diabetes remained significant but was attenuated (aHR $=1.69[95 \%$ CI $\left.1.47,1.94] I^{2}=62 \%\right)$ compared with studies that did not control for BMI (aHR = 2.71 [95\% CI 2.26, 3.25] $I^{2}=95 \%$ ). The effect of HDP on diabetes risk was also attenuated in studies that controlled for all of GDM, BMI, chronic hypertension, and history of CVD or cardiovascular risk factors together (aHR 1.89 [95\% CI $1.17,3.04] I^{2}=82 \%$ ).

Assessment of methodological quality Methodological quality varied across studies (ESM Table 3). Overall, 12 studies 


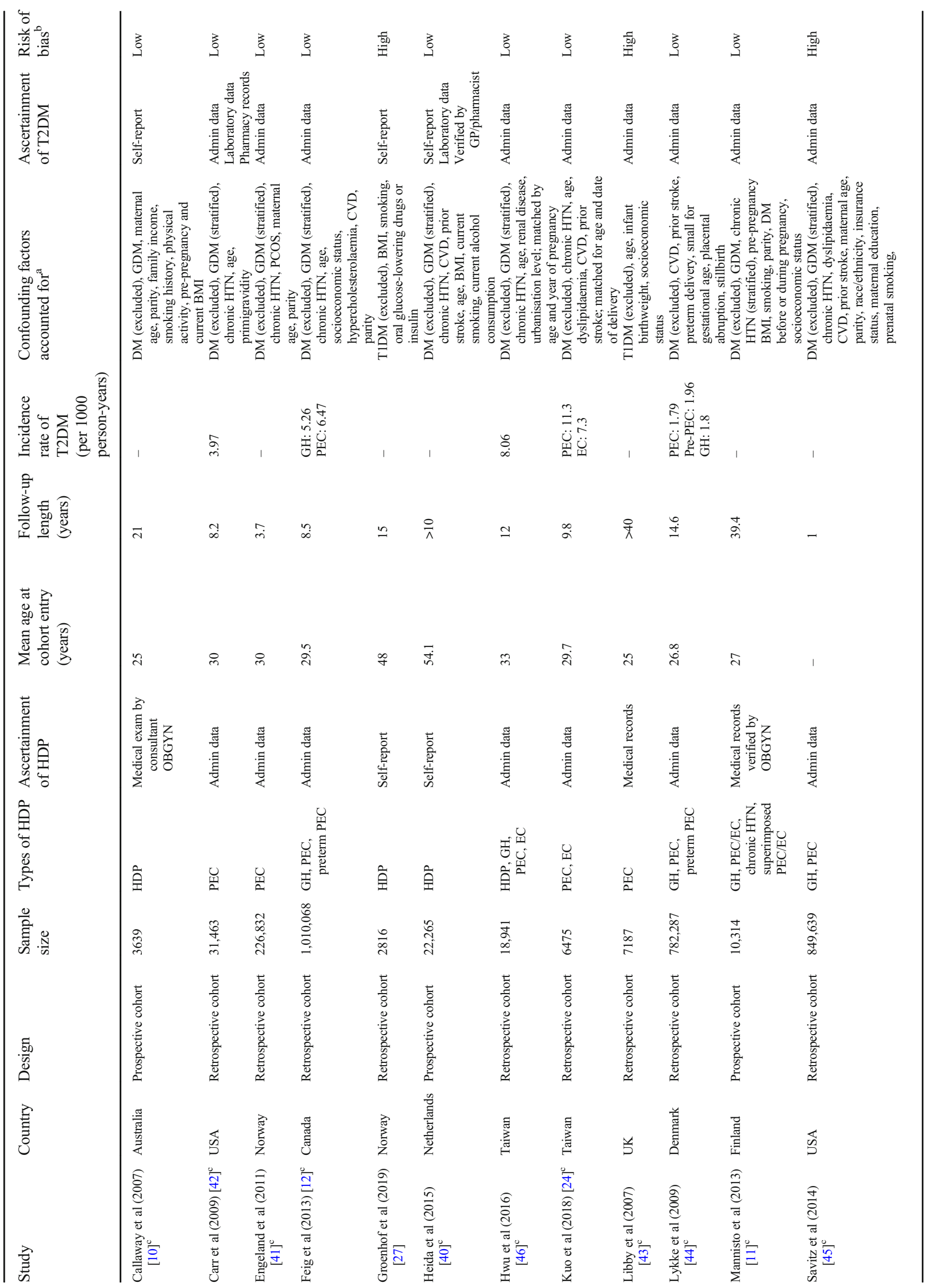




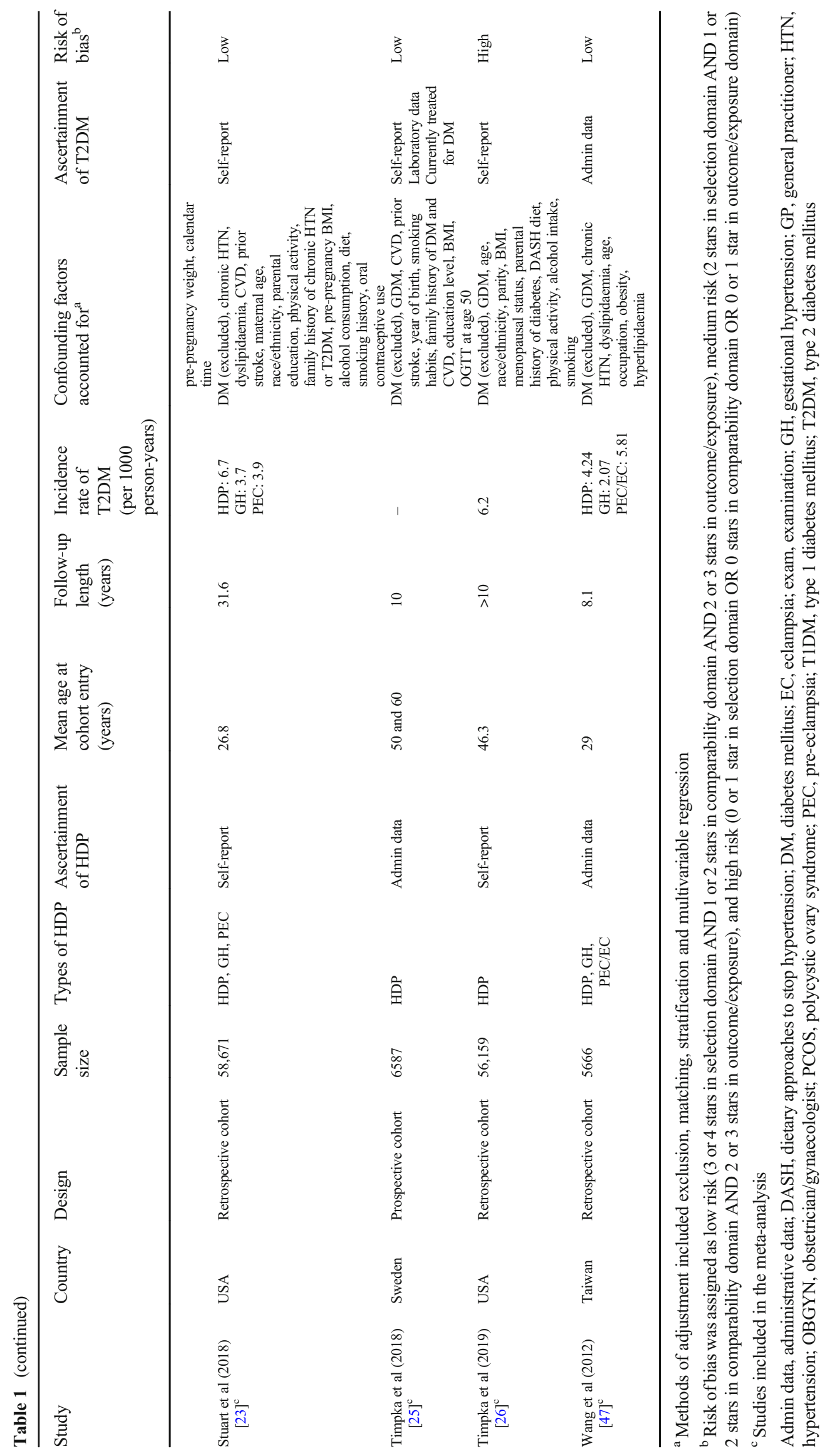


Table 2 Sensitivity and subgroup analyses examining the influence of follow-up duration and selected confounding factors

\begin{tabular}{|c|c|c|c|}
\hline Sensitivity analysis & Studies $(n)$ & $\operatorname{aHR}(95 \% \mathrm{CI})$ & $I^{2}(\%)$ \\
\hline \multicolumn{4}{|c|}{ Hypertensive disorder type } \\
\hline HDP (all) & 15 & $2.24(1.95,2.58)$ & 94 \\
\hline $\mathrm{GH}$ & 7 & $2.19(1.69,2.84)$ & 87 \\
\hline PEC & 11 & $2.56(2.02,3.24)$ & 94 \\
\hline Preterm PEC & 3 & $3.05(2.05,4.56)$ & 82 \\
\hline \multicolumn{4}{|l|}{ Controlled for GDM ${ }^{\mathrm{a}}$} \\
\hline HDP (all) & 11 & $2.01(1.77,2.28)$ & 85 \\
\hline $\mathrm{GH}$ & 5 & $2.01(1.54,2.63)$ & 80 \\
\hline PEC & 7 & $2.38(1.92,2.96)$ & 80 \\
\hline \multicolumn{4}{|c|}{ Controlled for chronic HTN } \\
\hline HDP (all) & 9 & $2.29(1.91,2.74)$ & 91 \\
\hline $\mathrm{GH}$ & 5 & $2.19(1.59,3.01)$ & 72 \\
\hline PEC & 7 & $3.03(2.33,3.94)$ & 75 \\
\hline \multicolumn{4}{|l|}{ Controlled for BMI } \\
\hline HDP (all) & 8 & $1.69(1.47,1.94)$ & 62 \\
\hline GH & 4 & $1.57(1.29,1.92)$ & 0 \\
\hline PEC & 4 & $2.32(1.46,3.68)$ & 71 \\
\hline \multicolumn{4}{|c|}{ Controlled for CVD risk factors } \\
\hline HDP (all) & 8 & $2.40(2.02,2.85)$ & 95 \\
\hline $\mathrm{GH}$ & 5 & $2.18(1.57,3.01)$ & 85 \\
\hline PEC & 6 & $3.10(2.20,4.37)$ & 96 \\
\hline \multicolumn{4}{|c|}{ Controlled for BMI, GDM, chronic HTN, CVD risk factors } \\
\hline HDP (all) & 3 & $1.89(1.17,3.04)$ & 82 \\
\hline $\mathrm{GH}$ & 2 & - & - \\
\hline PEC & 2 & - & - \\
\hline \multicolumn{4}{|c|}{ Individual follow-up duration ${ }^{\mathrm{b}}$} \\
\hline$<20$ years & 7 & $2.64(2.23,3.12)$ & 95 \\
\hline$\geq 20$ years & 2 & $1.34(1.20,1.50)$ & 0 \\
\hline
\end{tabular}

All included studies in the meta-analysis have controlled for baseline diabetes mellitus

$\mathrm{aHR}=$ pooled adjusted hazard ratios, reference groups $=$ no history of HDP

${ }^{\text {a }}$ Studies that controlled for GDM either by exclusion or regression

${ }^{\mathrm{b}}$ Studies that followed women from the time of delivery (mean age: 28.2 years, range: $25-33$ years)

GH, gestational hypertension; HTN, hypertension; PEC, pre-eclampsia

were rated to be of low risk of bias [10-12, 23-25, 40-42, 44, $46,47]$, while four studies were rated to be of high risk of bias $[26,27,43,45]$. In addition, five studies either did not adjust or were ambiguous about whether they adjusted for GDM [23, $24,27,43,44]$. Seven studies did not explicitly exclude chronic hypertension [10, 11, 25-27, 43, 44], although Mannisto et al (2013) reported the effects of HDP and chronic hypertension separately [11]. Eight studies controlled for the presence of other pre-pregnancy cardiovascular risk factors (e.g. history of hypercholesterolaemia, myocardial infarction, or stroke) [12, 23-25, 40, 44, 45, 47]. Four studies did not specify whether the outcome of diabetes included both type 1 and type 2 diabetes mellitus [24, 41, 42, 46]. Libby et al (2007) in particular had many potential confounding factors as medical records from the 1950s and 1960s lacked pertinent confounding information, such as personal and family history of diabetes and GDM [43]. Furthermore, their control group may have included pre-eclampsia cases from earlier pregnancies, which may have attenuated the estimated association between pre-eclampsia and diabetes due to information bias. Stuart et al (2018) adjusted for the largest number of possible confounders, including pre-pregnancy metabolic and behavioural risk factors [23]. There was a trend that more recent studies controlled for cardiovascular risk factors.

The funnel plot was symmetrically distributed about the pooled effect size estimate (Fig. 3) and Egger's test of the intercept was not significant $(p=0.6609)$. This suggests that publication bias is unlikely to play a major role in the interpretation of findings, though it cannot be ruled out completely, as high between-study heterogeneity in meta-analyses of observational studies may contribute to the observed funnel plot symmetry [32].

\section{Discussion}

This systematic review and meta-analysis of 16 studies with more than 3 million women showed that women with a history of HDP had a twofold increased risk of developing diabetes that is independent of BMI and GDM. There was a doseresponse relationship between HDP severity and diabetes risk, with pre-eclampsia/preterm pre-eclampsia having the greatest risk. Furthermore, the risk of diabetes associated with HDP was higher in studies with shorter follow-up (less than 20 years), suggesting that diabetes risk increases earlier in women with a history of HDP.

Two previous meta-analyses have been conducted on the risk of diabetes after HDP or pre-eclampsia [21, 22]. Our estimate of the association between any type of HDP and diabetes was higher than that from the meta-analysis of Wang et al [21]. Differences in our estimates are likely to be a result of (1) exclusion of studies with pre-gestational diabetes and (2) inclusion of studies published since 2017. Our estimates for the association between pre-eclampsia and subsequent diabetes are comparable to the meta-analysis of $\mathrm{Wu}$ et al [22]. However, we considered the influence of important confounding factors, such as BMI, GDM and cardiovascular risk factors more comprehensively. Additionally, we included more studies that had a length of follow-up greater than 10 years and found that among women who participated in such studies, the risk of diabetes was greatest at age 35-40 years. Further, several studies have found a relationship between HDP and future CVD [22, 23, 27, 48-51]. A large population-based retrospective cohort 
Study

Feig et al (2013) [12]

Lykke et al (2009) [44]

Mannisto et al (2013) [11]

Savitz et al (2014) [45]

Callaway et al (2007) [10]

Heida et al (2015) [40]

Hwu et al (2016) [46]

Stuart et al (2018) (full-term) [23]

Stuart et al (2018) (preterm) [23]

Timpka et al (2018) [25]

Timpka et al (2019) [26]

Wang et al (2012) [47]

Carr et al (2009) [42]

Engeland et al (2011) [41]

Feig et al (2013) [12]

Kuo et al (2018) [24]

Libby et al (2007) [43]

Lykke et al (2009) [44]

Mannisto et al (2013) [11]

Savitz et al (2014) [45]

Feig et al (2013) [12]

Kuo et al (2018) [24]

Lykke et al (2009) [44]
HDP type log[HR] SE

$\begin{array}{ccc}\text { GH } & 0.67 & 0.03 \\ \mathrm{GH} & 1.14 & 0.09 \\ \mathrm{GH} & 0.42 & 0.12 \\ \mathrm{GH} & 0.47 & 0.34 \\ \mathrm{HDP} & 0.57 & 0.19 \\ \mathrm{HDP} & 0.24 & 0.07 \\ \mathrm{HDP} & 1.15 & 0.11 \\ \mathrm{HDP} & 0.57 & 0.05 \\ \mathrm{HDP} & 0.49 & 0.12 \\ \mathrm{HDP} & 0.67 & 0.21 \\ \mathrm{HDP} & 0.73 & 0.34 \\ \mathrm{HDP} & 1.23 & 0.25 \\ \mathrm{PEC} & 0.62 & 0.22 \\ \mathrm{PEC} & 1.10 & 0.10 \\ \text { PEC } & 0.73 & 0.03 \\ \text { PEC } & 1.69 & 0.15 \\ \text { PEC } & 0.34 & 0.11 \\ \text { PEC } & 1.26 & 0.04 \\ \text { PEC } & 0.35 & 0.22 \\ \text { PEC } & 0.69 & 0.24 \\ \text { Preterm } & 0.80 & 0.12 \\ \text { Preterm } & 1.35 & 0.34 \\ \text { Preterm } & 1.30 & 0.10\end{array}$

HR

$[95 \% \mathrm{Cl}]$ Weight \%

(1)

$1.95 \quad[1.83 ; 2.07] \quad 5.5$

$3.12[2.63 ; 3.70] \quad 5.1$

$1.52[1.21 ; 1.90] \quad 4.9$

$1.60 \quad[0.82 ; 3.12] \quad 2.4$

$1.76[1.21 ; 2.56] \quad 4.0$

$1.27 \quad[1.11 ; 1.46] \quad 5.3$

$3.15[2.55 ; 3.89] \quad 4.9$

$1.76 \quad[1.61 ; 1.93] \quad 5.4$

$1.64[1.30 ; 2.07] \quad 4.8$

$1.96 \quad[1.29 ; 2.98] \quad 3.7$

$2.08 \quad[1.06 ; 4.07] \quad 2.4$

$3.42[2.08 ; 5.63] \quad 3.3$

$1.86 \quad[1.22 ; 2.84] \quad 3.7$

$3.00[2.46 ; 3.66] \quad 5.0$

$2.08 \quad[1.97 ; 2.19] \quad 5.5$

$5.42 \quad[4.01 ; 7.32] \quad 4.4$

$1.40[1.12 ; 1.75] \quad 4.9$

$3.53[3.23 ; 3.85] \quad 5.4$

$1.42[0.92 ; 2.19] \quad 3.6$

$2.00[1.26 ; 3.17] \quad 3.5$

$2.23[1.77 ; 2.81] \quad 4.8$

$3.84[1.99 ; 7.40] \quad 2.5$

$3.68[3.04 ; 4.46] \quad 5.0$

$2.24[1.95 ; 2.58] 100.0 \%$

Overall effect

Heterogeneity: $I^{2}=94 \%[92 \% ; 95 \%], \tau^{2}=0.0913, p<0.01$

Fig. 2 Forest plot for risk of diabetes in women with prior HDP. Effect size estimates depicted to the left of the overall effect represent a lower risk of diabetes in those with HDP, while effect sizes to the right of the overall effect represent a higher risk of diabetes in those with HDP. The values in the ' $\log [\mathrm{HR}]$ ' column represent natural logarithms (i.e. $\log$ to the base of e) for HR estimates from each of the included studies. 'Preterm' denotes pre-eclampsia that necessitated preterm delivery (<37 weeks). GH, gestational hypertension; PEC, pre-eclampsia

early-onset pre-eclampsia as a distinct disease entity [53, 54]. Therefore, our study provides further evidence in favour of an independent association between HDP and subsequent diabetes.

The strength of our study lies in the number of recent studies included, the large sample size, accounting for key confounders such as GDM status, BMI and cardiovascular
Fig. 3 Funnel plot for the assessment of publication bias in the meta-analysis on HDP (all studies). The $y$-axis represents natural logarithms (i.e. log to the base of e) for HR estimates from each of the included studies. The $x$-axis is plotted on a log scale. The dotted lines represent the pooled random-effects HR estimate with $95 \%$ CI

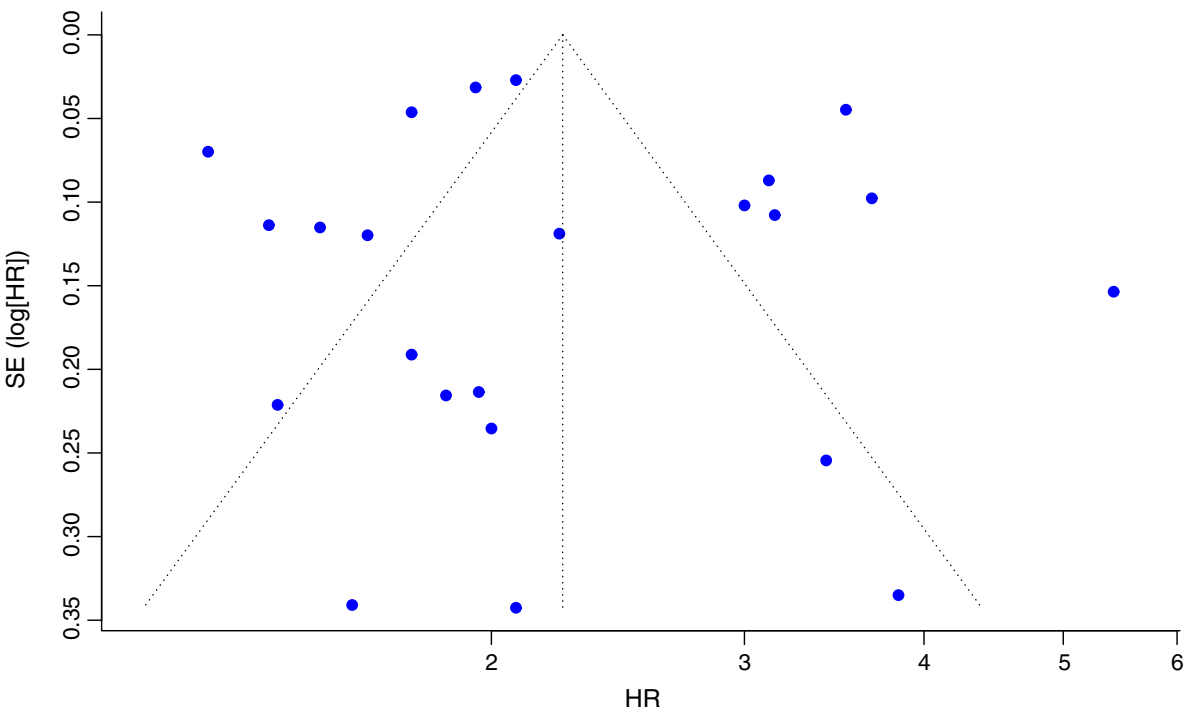


risk factors, and the influence of time since delivery on diabetes risk. We employed a comprehensive search strategy and a rigorous review process.

Several limitations were identified in our study. As the majority of included studies were retrospective studies, there is limitation in the quality of data collected. A number of studies used self-report to determine either the exposure of HDP or the outcome of diabetes. There was also significant heterogeneity in the meta-analysis which can be partially attributed to variability in study designs and quality ratings, as well as the inherent vulnerability of metaanalyses of observational studies to residual confounding. Other important confounding factors such as family history of diabetes [55], ethnicity [56], lifestyle (diet, physical activity, smoking) [57] and recurrent HDP [58] were seldom adjusted for. Further, the risk estimates from Taiwanese studies [24, 46, 47] may have amplified the association between HDP and diabetes due to higher insulin resistance in Asian women [59]. Only a limited number of studies included information on the age at diabetes diagnosis; however, age at pregnancy and follow-up duration were more consistently reported, allowing us to estimate when the risk of diabetes may be the highest. The high variability of HDP prevalence may also be indicative of selection bias in some studies. Nonetheless, the higher prevalence of HDP in some study samples may bias our findings towards the null; as such, the conclusion that diabetes risk is elevated among women with prior HDP would still hold true. Finally, random-effects meta-analyses award relatively more weight to smaller, and potentially underpowered, studies than fixed-effects models [32]. The DerSimonianLaird method may also underestimate confidence intervals and lead to erroneous conclusions about the statistical significance of findings when the meta-analysis sample size is small and statistical heterogeneity is high [60,61]. Subgroup analyses of fewer than four studies should thus be interpreted with caution [62].

The exact mechanisms between HDP and future diabetes are unknown. It has been hypothesised that insulin resistance may play a role in the disease pathogenesis of new-onset hypertension in pregnancy [63], which may explain the persistence of metabolic abnormalities after delivery and subsequent diabetes. Similar to GDM, HDP may be an earlier manifestation of underlying insulin resistance that is brought out by the increased metabolic demands of pregnancy [9, 22, 63]. HDP share many risk factors with CVD: obesity, hyperlipidaemia, hypertension, insulin resistance and renal dysfunction $[22$, 64]. HDP also co-occur with GDM in about $4 \%$ of pregnancies [65], which is a known strong risk factor for subsequent diabetes [12, 15-18]. Our study showed that HDP increase the risk of incident diabetes independent of BMI and GDM, suggesting an additive effect of HDP on diabetes risk. This may be related to abnormal cytotrophoblast invasion of spiral arterioles leading to reduced placental perfusion and subsequent maternal vascular endothelium dysfunction, which is associated with upregulated levels of the antiangiogenic placental soluble fms-like tyrosine kinase $1[66,67]$. This suggests that the presence of HDP may worsen pre-existing metabolic risk factors or even induce de novo risk [68]. In fact, a large population-based study demonstrated that most cardiovascular risk factors remained significantly elevated after preeclampsia following adjustment for pre-pregnancy values [69]. Additionally, recent evidence has shown a link between HDP and future risk of chronic kidney disease that is independent of GDM [70]. This further suggests that type 2 diabetes may be an intermediate factor between HDP and other cardiometabolic diseases.

Implications of findings and future directions The time lag between HDP and the onset of diabetes presents a window of opportunity for timely interventions. Subclinical atherosclerosis is visible by cardiovascular imaging by 16 years postpartum for women with a history of pre-eclampsia [71]. Some guidelines advise follow-up of women with HDP 6 8 weeks postpartum [72,73], and others recommend 6 12 months postpartum [74]. For diabetes specifically, current screening guidelines do not include HDP as an independent risk factor; as such, screening would not be recommended until the age of $40[16,75,76]$.

As has been recommended for the prevention of cardiovascular outcomes [77], counselling regarding diabetes risk may be warranted in women with pregnancies complicated by HDP. Lifestyle modification and weight management should be prioritised, as elevated BMI may have a synergistic effect with HDP on diabetes risk [26]. Such interventions may also decrease their risk of recurrent HDP in future pregnancies.

There is a lack of evidence regarding diabetes risk stratification and postpartum screening for women who experience adverse pregnancy outcomes beyond GDM [78]. While earlier screening for diabetes may be warranted among women with HDP, our findings highlight the need for primary evidence regarding how HDP contribute to diabetes risk prediction to develop evidence-based screening and prevention strategies.

Conclusion This systematic review and meta-analysis showed that HDP are independently associated with a twofold increased risk of subsequent diabetes. Postpartum diabetes screening and prevention counselling may be warranted in women with a history of HDP, even in the absence of GDM. Primary evaluative evidence is necessary to determine the optimal follow-up strategy targeted to women with a history of HDP.

Supplementary Information The online version contains peer-reviewed but unedited supplementary material available at https://doi.org/10.1007/ s00125-020-05343-w. 
Acknowledgements We would like to thank E. Springall (Gerstein Science Information Centre, University of Toronto) for her guidance in the design of the electronic search strategy. Author L. Lipscombe is supported by a Diabetes Investigator Award from Diabetes Canada.

Data availability The protocol can be accessed on PROSPERO (registration ID CRD42020168399). Data can be accessed upon request by emailing the corresponding author (grace.zhao@mail.utoronto.ca). Reuse only with permission and with citation.

Funding This research received no specific grant from any funding agency in the public, commercial or not-for-profit sectors.

Authors' relationships and activities The authors declare that there are no relationships or activities that might bias, or be perceived to bias, their work.

Contribution statement GZ and LL conceptualised and designed the study. GZ, DB and FJ performed data collection and risk of bias appraisal. DB performed the meta-analysis. GZ performed data abstraction, narrative synthesis and drafting of the first version of the manuscript. All authors contributed critically to interpretation of the data and manuscript revisions. All authors approved the final version to be published. GZ and LL had full access to the data in the study and take responsibility for the integrity of the data and accuracy of the analysis. The corresponding author attests that all listed authors meet authorship criteria.

\section{References}

1. Fraser A, Nelson SM, Macdonald-Wallis C et al (2012) Associations of pregnancy complications with calculated cardiovascular disease risk and cardiovascular risk factors in middle age: the Avon Longitudinal Study of Parents and Children . Circulation 125(11):1367-1380. https://doi.org/10.1161/ CIRCULATIONAHA.111.044784

2. Umesawa M, Kobashi G (2017) Epidemiology of hypertensive disorders in pregnancy: prevalence, risk factors, predictors and prognosis. Hypertens Res 40(3):213-220. https://doi.org/10.1038/ hr.2016.126

3. ACOG (2020) Practice bulletin no. 222: gestational hypertension and preeclampsia. Obstet Gynecol 135(6):e237-e260. https://doi. org/10.1097/AOG.0000000000003891

4. Bethesda M (2000) Report of the National High Blood Pressure Education Program Working Group on High Blood Pressure in Pregnancy. Am J Obstet Gynecol 183(1):s1-s22. https://doi.org/ 10.1067/mob.2000.107928

5. Pouta A, Hartikainen A-L, Sovio U et al (2004) Manifestations of metabolic syndrome after hypertensive pregnancy. Hypertension 43(4):825-831. https://doi.org/10.1161/01.HYP.0000120122. 39231.88

6. Parretti E, Lapolla A, Dalfra M et al (2006) Preeclampsia in lean normotensive normotolerant pregnant women can be predicted by simple insulin sensitivity indexes. Hypertension 47(3):449-453. https://doi.org/10.1161/01.HYP.0000205122.47333.7f

7. Sierra-Laguado J, Garcia RG, Celedon J et al (2007) Determination of insulin resistance using the homeostatic model assessment (HOMA) and its relation with the risk of developing pregnancyinduced hypertension. Am J Hypertens 20(4):437-442

8. Carpenter MW (2007) Gestational diabetes, pregnancy hypertension, and late vascular disease. Diabetes Care 30(Supplement 2): S246. https://doi.org/10.2337/dc07-s224
9. Viana Pinto P, Rei M, Machado AP, Montenegro N (2014) Preeclampsia and future cardiovascular risk: are women and general practitioners aware of this relationship? The experience from a Portuguese centre. Obstet Gynecol Int 2014. https://doi.org/10. $1155 / 2014 / 531539$

10. Callaway LK, Lawlor DA, O'Callaghan M, Williams GM, Najman JM, McIntyre HD (2007) Diabetes mellitus in the 21 years after a pregnancy that was complicated by hypertension: findings from a prospective cohort study. Am J Obstet Gynecol 197(5):492.e1-7. https://doi.org/10.1016/j.ajog.2007.03.033

11. Mannisto T, Mendola P, Vaarasmaki M et al (2013) Elevated blood pressure in pregnancy and subsequent chronic disease risk. Circulation 127(6):681-690. https://doi.org/10.1161/ CIRCULATIONAHA.112.128751

12. Feig DS, Shah BR, Lipscombe LL et al (2013) Preeclampsia as a risk factor for diabetes: a population-based cohort study. PLOS Med 10(4):e1001425. https://doi.org/10.1371/journal.pmed. 1001425

13. Bilandzic A, Rosella L (2017) The cost of diabetes in Canada over 10 years: applying attributable health care costs to a diabetes incidence prediction model. Health Promotion and Chronic Disease Prevention in Canada Health Promot Chronic Dis Prev Can 37(2): 49-53. https://doi.org/10.24095/hpcdp.37.2.03

14. Saeedi P, Petersohn I, Salpea P et al (2019) Global and regional diabetes prevalence estimates for 2019 and projections for 2030 and 2045: results from the International Diabetes Federation Diabetes Atlas, 9th edition. Diabetes Research and Clinical Practice 157. https://doi.org/10.1016/j.diabres.2019.107843

15. Feig DS, Zinman B, Wang X, Hux JE (2008) Risk of development of diabetes mellitus after diagnosis of gestational diabetes. CMAJ 179(3):229-234. https://doi.org/10.1503/cmaj.080012

16. Canada D (2018) Diabetes Canada 2018 clinical practice guidelines for the prevention and management of diabetes in Canada. Can J Diabetes 42(Suppl 1):S1-S325

17. Noctor E, Dunne FP (2015) Type 2 diabetes after gestational diabetes: the influence of changing diagnostic criteria. World J Diabetes 6(2):234-244. https://doi.org/10.4239/wjd.v6.i2.234

18. Bellamy L, Casas J-P, Hingorani AD, Williams D (2009) Type 2 diabetes mellitus after gestational diabetes: a systematic review and meta-analysis. Lancet 373(9677):1773-1779. https://doi.org/10. 1016/S0140-6736(09)60731-5

19. Association AD (2003) Screening for type 2 diabetes. Diabetes Care 26(supp1 1):s21-s24. https://doi.org/10.2337/diacare.26. 2007.S21

20. NICE (2012) Type 2 diabetes: prevention in people at high risk (Public health guideline PH38). Available from https://www.nice. org.uk/guidance/ph38/evidence. Accessed Apr 2020

21. Wang Z, Wang Z, Wang L et al (2017) Hypertensive disorders during pregnancy and risk of type 2 diabetes in later life: a systematic review and meta-analysis. Endocrine 55(3):809-821. https:// doi.org/10.1007/s12020-016-1075-6

22. Wu P, Kwok CS, Haththotuwa R et al (2016) Pre-eclampsia is associated with a twofold increase in diabetes: a systematic review and meta-analysis. Diabetologia 59(12):2518-2526. https://doi.org/ 10.1007/s00125-016-4098-x

23. Stuart JJ, Tanz LJ, Missmer SA et al (2018) Hypertensive disorders of pregnancy and maternal cardiovascular disease risk factor development: an observational cohort study. Ann Intern Med 169(4): 224-232. https://doi.org/10.7326/m17-2740

24. Kuo Y-L, Chan T-F, Wu C-Y, Ker C-R, Tu H-P (2018) Preeclampsia-eclampsia and future cardiovascular risk among women in Taiwan. Taiwan J Obstet Gynecol 57(3):364-369. https://doi.org/10.1016/j.tjog.2018.04.035

25. Timpka S, Markovitz A, Schyman T et al (2018) Midlife development of type 2 diabetes and hypertension in women by history of 
hypertensive disorders of pregnancy. Cardiovasc Diabetol 17(1): 124. https://doi.org/10.1186/s12933-018-0764-2

26. Timpka S, Stuart JJ, Tanz LJ, Hu FB, Franks PW, Rich-Edwards JW (2019) Postpregnancy BMI in the progression from hypertensive disorders of pregnancy to type 2 diabetes. Diabetes Care 42(1): 44-49. https://doi.org/10.2337/dc18-1532

27. Groenhof TKJ, Zoet GA, Franx A et al (2019) Trajectory of cardiovascular risk factors after hypertensive disorders of pregnancy. Hypertension 73(1):171-178. https://doi.org/10.1161/ HYPERTENSIONAHA.118.11726

28. Moher D, Liberati A, Tetzlaff J, Altman DG, The PG (2009) Preferred reporting items for systematic reviews and meta-analyses: the PRISMA statement. PLoS Med 6(7):e1000097. https://doi.org/ 10.1371/journal.pmed.1000097

29. Liberati A, Altman DG, Tetzlaff J et al (2009) The PRISMA statement for reporting systematic reviews and meta-analyses of studies that evaluate health care interventions: explanation and elaboration. PLoS Med 6(7):e1000100. https://doi.org/10.1371/journal.pmed. 1000100

30. Stroup DF, Berlin JA, Morton SC et al (2000) Meta-analysis of observational studies in epidemiology: a proposal for reporting. JAMA 283(15):2008-2012. https://doi.org/10.1001/jama.283.15. 2008

31. BMJ Best Practice (2019) Study design search filters. Available from https://bestpractice.bmj.com/info/toolkit/learn-ebm/studydesign-search-filters/. Accessed Feb 2020

32. Higgins JPT TJ, Chandler J, Cumpston M, Li T, Page MJ, Welch VA (2019) Cochrane Handbook for systematic reviews of interventions (updated July 2019). Available from www.training.cochrane. org/handbook. Accessed 5 Feb 2020

33. Reeves BCDJ, Higgins JPT, Wells GA (2008) Chapter 13: Including non-randomized studies. In: Higgins JPTGS (ed) Cochrane handbook for systematic reviews of interventions. Wiley, Chichester

34. Wells G, Shea B, O'Connell D et al (2000) The Newcastle-Ottawa Scale (NOS) for assessing the quality of non-randomized studies in meta-analysis. Available from http://www.ohri.ca/programs/ clinical_epidemiology/oxford.asp. Accessed 1 Apr 2020

35. DerSimonian R, Laird N (1986) Meta-analysis in clinical trials. Control Clin Trials 7(3):177-188. https://doi.org/10.1016/01972456(86)90046-2

36. Zhang J, Yu KF (1998) What's the relative risk? A method of correcting the odds ratio in cohort studies of common outcomes. Jama 280(19):1690-1691. https://doi.org/10.1001/jama.280.19. 1690

37. Wilmot EG, Edwardson CL, Achana FA et al (2012) Sedentary time in adults and the association with diabetes, cardiovascular disease and death: systematic review and meta-analysis. Diabetologia 55(11):2895-2905. https://doi.org/10.1007/s00125012-2677-z

38. Biswas A, Oh PI, Faulkner GE et al (2015) Sedentary time and its association with risk for disease incidence, mortality, and hospitalization in adults: a systematic review and meta-analysis. Ann Intern Med 162(2):123-132. https://doi.org/10.7326/m14-1651

39. Sutradhar R, Austin PC (2018) Relative rates not relative risks: addressing a widespread misinterpretation of hazard ratios. Ann Epidemiol 28(1):54-57. https://doi.org/10.1016/j.annepidem. 2017.10.014

40. Heida KY, Franx A, Rijn BBV et al (2015) Earlier age of onset of chronic hypertension and type 2 diabetes mellitus after a hypertensive disorder of pregnancy or gestational diabetes mellitus. Hypertension 66(6):1116-1122. https://doi.org/10.1161/ HYPERTENSIONAHA.115.06005

41. Engeland A, Bjørge T, Daltveit AK et al (2011) Risk of diabetes after gestational diabetes and preeclampsia. A registry-based study of 230,000 women in Norway. Eur J Epidemiol 26(2):157-163. https://doi.org/10.1007/s10654-010-9527-4

42. Carr DB, Newton KM, Utzschneider KM et al (2009) Preeclampsia and risk of developing subsequent diabetes. Hypertens Pregnancy 28(4):435-447. https://doi.org/10.3109/10641950802629675

43. Libby G, Murphy DJ, McEwan NF et al (2007) Pre-eclampsia and the later development of type 2 diabetes in mothers and their children: an intergenerational study from the Walker cohort. Diabetologia 50(3):523-530. https://doi.org/10.1007/s00125-0060558-z

44. Lykke JA, Langhoff-Roos J, Sibai BM, Funai EF, Triche EW, Paidas MJ (2009) Hypertensive pregnancy disorders and subsequent cardiovascular morbidity and type 2 diabetes mellitus in the mother. Hypertension 53(6):944-951. https://doi.org/10.1161/ HYPERTENSIONAHA.109.130765

45. Savitz DA, Danilack VA, Elston B, Lipkind HS (2014) Pregnancyinduced hypertension and diabetes and the risk of cardiovascular disease, stroke, and diabetes hospitalization in the year following delivery. Am J Epidemiol 180(1):41-44. https://doi.org/10.1093/ aje/kwu118

46. Hwu L-J, Sung F-C, Mou C-H et al (2016) Risk of subsequent hypertension and diabetes in women with hypertension during pregnancy and gestational diabetes. Mayo Clin Proc 91(9):11581165. https://doi.org/10.1016/j.mayocp.2016.05.017

47. Wang IK, Tsai IJ, Chen P-C et al (2012) Hypertensive disorders in pregnancy and subsequent diabetes mellitus: a retrospective cohort study. Am J Med 125(3):251-257. https://doi.org/10.1016/j. amjmed.2011.07.040

48. Bellamy L, Casas J-P, Hingorani AD, Williams DJ (2007) Preeclampsia and risk of cardiovascular disease and cancer in later life: systematic review and meta-analysis. BMJ 335(7627):974-974. https://doi.org/10.1136/bmj.39335.385301.BE

49. Hannaford P, Ferry S, Hirsch S (1997) Cardiovascular sequelae of toxaemia of pregnancy. Heart 77(2):154-158. https://doi.org/10. $1136 /$ hrt.77.2.154

50. Kestenbaum B, Seliger SL, Easterling TR et al (2003) Cardiovascular and thromboembolic events following hypertensive pregnancy. Am J Kidney Dis 42(5):982-989. https://doi.org/ 10.1016/j.ajkd.2003.07.001

51. Srivaratharajah K, Abramson BL (2019) Identifying and managing younger women at high risk of cardiovascular disease. CMAJ 191(6):E159-E163. https://doi.org/10.1503/cmaj.180053

52. Ray JG, Vermeulen MJ, Schull MJ, Redelmeier DA (2005) Cardiovascular health after maternal placental syndromes (CHAMPS): population-based retrospective cohort study. Lancet 366(9499):17971803. https://doi.org/10.1016/S0140-6736(05)67726-4

53. Vatten LJ, Skjærven R (2004) Is pre-eclampsia more than one disease? BJOG Int J Obstet Gynaecol 111(4):298-302. https://doi. org/10.1111/j.1471-0528.2004.00071.x

54. Raymond DPE (2011) Critical review of early-onset and late-onset preeclampsia. Obstet Gynecol Surv 66(8):497-506. https://doi.org/ 10.1097/OGX.0b013e3182331028

55. Scott RA, Langenberg C, Sharp SJ et al (2013) The link between family history and risk of type 2 diabetes is not explained by anthropometric, lifestyle or genetic risk factors: the EPIC-InterAct study. Diabetologia 56(1):60-69. https://doi.org/10.1007/s00125-012-2715-x

56. Spanakis EK, Golden SH (2013) Race/ethnic difference in diabetes and diabetic complications. Curr Diab Rep 13(6):814-823. https:// doi.org/10.1007/s11892-013-0421-9

57. Ghaderpanahi M, Fakhrzadeh H, Sharifi F et al (2011) Association of physical activity with risk of type 2 diabetes. Iran J Public Health 40(1):86-93

58. Brouwers L, van der Meiden-van Roest A, Savelkoul C et al (2018) Recurrence of pre-eclampsia and the risk of future hypertension and cardiovascular disease: a systematic review and meta-analysis. 
BJOG Int J Obstet Gynaecol 125(13):1642-1654. https://doi.org/ 10.1111/1471-0528.15394

59. Kong APS, Luk AOY, Chan JCN (2016) Detecting people at high risk of type 2 diabetes - how do we find them and who should be treated? Best Pract Res Clin Endocrinol Metab 30(3):345-355. https://doi.org/10.1016/j.beem.2016.06.003

60. Cornell J, Mulrow C, Localio R et al (2014) Random-effects metaanalysis of inconsistent effects: a time for change. Ann Intern Med 160(4):267-270. https://doi.org/10.7326/m13-2886\%m24727843

61. IntHout J, Ioannidis JPA, Borm GF (2014) The Hartung-KnappSidik-Jonkman method for random effects meta-analysis is straightforward and considerably outperforms the standard DerSimonianLaird method. BMC Med Res Methodol 14:25-25. https://doi.org/ 10.1186/1471-2288-14-25

62. Bender R, Friede T, Koch A et al (2018) Methods for evidence synthesis in the case of very few studies. Res Synth Methods 9(3):382-392. https://doi.org/10.1002/jrsm.1297

63. Seely EW, Solomon CG (2003) Insulin resistance and its potential role in pregnancy-induced hypertension. J Clin Endocrinol Metab 88(6):2393-2398. https://doi.org/10.1210/jc.2003-030241

64. Hashemi S, Ramezani Tehrani F, Mehrabi Y, Azizi F (2013) Hypertensive pregnancy disorders as a risk factor for future cardiovascular and metabolic disorders (Tehran Lipid and Glucose Study). J Obstet Gynaecol Res 39(5):891-897. https://doi.org/10. 1111/j.1447-0756.2012.02069.x

65. Bryson CL, Ioannou GN, Rulyak SJ, Critchlow C (2003) Association between gestational diabetes and pregnancy-induced hypertension. Am J Epidemiol 158(12):1148-1153. https://doi.org/ 10.1093/aje/kwg273

66. Granger JP, Alexander BT, Bennett WA, Khalil RA (2001) Pathophysiology of pregnancy-induced hypertension. Am J Hypertens 14(S3):178S-185S. https://doi.org/10.1016/s08957061(01)02086-6

67. Maynard SE, Min J-Y, Merchan J et al (2003) Excess placental soluble fms-like tyrosine kinase 1 (sFlt1) may contribute to endothelial dysfunction, hypertension, and proteinuria in preeclampsia. J Clin Invest 111(5):649-658. https://doi.org/10.1172/JCI17189

68. Staff AC, Redman CWG, Williams D et al (2016) Pregnancy and long-term maternal cardiovascular health. Hypertension 67(2):251260. https://doi.org/10.1161/HYPERTENSIONAHA.115.06357

69. Romundstad PR, Magnussen EB, Smith GD, Vatten LJ (2010) Hypertension in pregnancy and later cardiovascular risk.
Circulation 122(6):579-584. https://doi.org/10.1161/ CIRCULATIONAHA.110.943407

70. Barrett PM, McCarthy FP, Kublickiene K et al (2020) Adverse pregnancy outcomes and long-term maternal kidney disease: a systematic review and meta-analysis. JAMA Netw Open 3(2): e1920964-e1920964. https://doi.org/10.1001/jamanetworkopen. 2019.20964

71. Zoet GA, Benschop L, Boersma E et al (2018) Prevalence of subclinical coronary artery disease assessed by coronary computed tomography angiography in 45- to 55-year-old women with a history of preeclampsia. Circulation 137(8):877-879. https://doi.org/10. 1161/CIRCULATIONAHA.117.032695

72. Visintin C, Mugglestone MA, Almerie MQ, Nherera LM, James D, Walkinshaw S (2010) Management of hypertensive disorders during pregnancy: summary of NICE guidance. BMJ 341:c2207. https://doi.org/10.1136/bmj.c2207

73. Mosca L, Benjamin EJ, Berra K et al (2011) Effectiveness-based guidelines for the prevention of cardiovascular disease in women2011 update. Circulation 123(11):1243-1262. https://doi.org/10. 1161/CIR.0b013e31820faaf8

74. Regitz-Zagrosek V, Roos-Hesselink JW, Bauersachs J et al (2018) 2018 ESC guidelines for the management of cardiovascular diseases during pregnancy: the task force for the management of cardiovascular diseases during pregnancy of the European Society of Cardiology (ESC). Eur Heart J 39(34):3165-3241. https://doi. org/10.1093/eurheartj/ehy340

75. Association AD (2002) Screening for diabetes. Diabetes Care 25(suppl 1):s21-s24. https://doi.org/10.2337/diacare.25.2007.S21

76. Karly Pippitt ML, Gurgle HE (2016) Diabetes mellitus: screening and diagnosis. Am Fam Physician 93(2):103-109

77. Benschop L, Duvekot JJ, Roeters van Lennep JE (2019) Future risk of cardiovascular disease risk factors and events in women after a hypertensive disorder of pregnancy. Heart 105(16):1273-1278. https://doi.org/10.1136/heartjnl-2018-313453

78. Barrett PM, Khashan AS, McCarthy FP, Kublickiene K (2020) Adverse pregnancy outcomes and maternal health: action needed for long-term benefit. Acta Obstetricia et Gynecologica Scandinavica n/a(n/a). https://doi.org/10.1111/aogs.13945

Publisher's note Springer Nature remains neutral with regard to jurisdictional claims in published maps and institutional affiliations. 